\title{
A new nine-node element for analysing plates with varying thickness using basic displacement functions
}

\author{
A.M. Haghighi ${ }^{1}$, K. Tayyebi $^{2}$, R. Attarnejad ${ }^{1,3}$ \\ ${ }^{1}$ School of Civil Engineering, College of Engineering, University of Tehran \\ 16th Azar St., Enghelab Sq., Tehran, Iran \\ ${ }^{2}$ Department of Civil Engineering, University of Victoria \\ 3800 Finnerty Road, Victoria, BC, Canada \\ Phone: +1(236)882-3922 \\ *Email: ktayyebi@uvic.ca \\ ${ }^{3}$ Centre of Numerical Methods in Engineering, \\ University of Tehran, Tehran, Iran
}

\begin{abstract}
The capability of the Finite Element Method in producing accurate and efficient results largely depends on the shape functions adopted to frame the displacement field inside the element. In this paper, a new nine-node Lagrangian element was developed to analyse thin plates with varying cross-sections using the shape functions obtained for nonprismatic straight beams with minimum number of elements. The formulated shape functions, which represent vertical displacements and rotations throughout elements, are rooted from a purely mechanical functions called Basic Displacement Functions (BDFs). These functions are obtained by implementing the force method in Euler-Bernoulli beam theory, which ensures that equilibrium equation is satisfied in all interior points of elements. To verify the competency of the proposed element, solutions for the static analysis of isotropic rectangular plates under various loading conditions, together with free vibration analysis of plates with linear thickness variation were obtained and compared with the previous literature. Results showed that the proposed nine-node Lagrangian element was computationally more cost-effective compared to other competing methods when small number of elements is employed.
\end{abstract}

Keywords: Basic Displacement Functions; Vibration analysis; Shape functions; EulerBernoulli beam theory.

\section{INTRODUCTION}

Rectangular shells and thin plates are among the most popular structural elements, which have extensive applications in the industry, from residential structures, bridge girders and decks to different mechanical usages such as aircraft components, automobile machinery, and storage tanks. The main advantage that light-weight thin plates provide is their relatively strong resistance against external forces which is mostly endured by their bilateral behaviour [1]. During the past century, the static and dynamic behaviour of plates has been the subject of vast investigations [2].

The first serious theory to analyse thin plates was proposed by Love [3] which was an extension of Euler-Bernoulli beam theory. He rewrote complicated formulations of threedimensional plates to a simpler two-dimensional form using simplifying Kirchhoff 
assumptions. Another significant contribution to the theory of plate elements was made by Reissner [4] who acknowledged the effect of transverse shear deformation on the behavior of relatively thick plates. Mindlin [5] then simplified Reissner's theory and slightly improved its assumption and expanded its application. Nonetheless, it is believed that introducing dynamic equation for vibration of plates by Lagrange was the starting point for further extensive investigations in this area. Moreover, collecting available classical solutions such as variational method, finite difference method and Rayleigh-Ritz method for solving problems associated with plates with different boundary conditions in the Leissa's technical report [6] substantially helped further investigations in the middle of the twentieth century. Despite extensive studies in this field, most published articles are concerned with the vibrational behaviour of plates with uniform thickness as the differential equations governing the vibration of plates of varying thickness are of higher order with variable coefficients. However, by a significant improvement in the plate manufacturing technologies, it is now possible to form plates with varying thickness effortlessly. The reduction of thickness in unnecessary parts of plates with lower stress concentrations not only decreases the cost of the structural element, but also reduces the weight of the element which plays a key role in some delicate applications such as satellite and aeronautical engineering. Therefore, it is mandatory to develop efficient methods to analyse the behaviour of plates with varying thickness similar to plates with uniform thickness. The efficient and systematic design of thin plate elements calls for static and stability, as well as dynamic analyses. In the case of static analyses, most of the conducted studies were mainly focused on buckling analysis of plates with generic loading and boundary conditions [7-10].

To date, many numerical methods have been proposed for finding mode shapes and frequencies of vibration of plates including Meshfree methods [11-13], LeastSquare-based Finite Difference method (LSFD) [14], Differential Quadrature Method (DQM) [15,16], discrete singular convolution method [17], Rayleigh-Ritz method [18], extended Kantorovich method [19], NURBS-based isogeometric method [20], and Finite Element Method (FEM) [21,22]. Kukreti et al. [23] found the fundamental frequency of thin elastic tapered plates with clamped, simply supported, and mixed boundary conditions [14]. Approximate solution for the free vibration analysis of rectangular plates with uniform and non-uniform thickness using Green function was offered by Sakiyama and Huang [24]. Ashur [25] used the finite strip transition matrix technique to study the free vibration analysis of plates with variable thickness assuming edges are elastically restrained against both rotation and transition. Application of power series expansion method for solving differential equation with variable coefficients governing the vibration of tapered plates is proposed by Kobayashi and Sonoda [26]. The main fashion of analysing plates with variable thickness is using finite element method as it is a general technique to model complex structures with various loading and boundary conditions. However, FEM is a computationally expensive tool to model nonlinearity, especially in case of complex geometries, where extremely small meshes are required to yield acceptable results. Hence, new and more efficient elements have been constantly formulated to reduce computation time, while they maintain or even improve the accuracy of results. The new elements introduced for analysing thin plates are either founded on novel ideas such as the least square method [27], Trefftz method [28], Wavelet-based method [29], isogeometric finite element method [20] or trying to improve the characteristics of existing elements [30,31]. Until now, the finite element method has been successfully employed in many finite element software packages to solve platerelated problems by discretising the entire domain into small meshes and imposing 
realistic boundary conditions. This method, however, is limited in that it can only explain the behaviour of plates of varying thickness with specific geometries, loading and boundary conditions. Even if enough care is given to model the principal factors such as geometry, loading, and boundary conditions as realistic as possible, yielding acceptable results call for implementing small and, in some cases, complex elements. This alone creates an inherent computational cost for solving problems such as general plates with varying thickness.

In an attempt to solve this issue, Basic displacement functions were introduced by Attarnejad [32]. The main difference of this approach in comparison to most finite element displacement-based formulations is its flexibility-based nature. However, compared to most solutions based on the force method, complicated mathematical formulations are not required to solve the equations. According to the definition, which is a pure mechanical one, the basic displacement functions can be derived as a function of displacement and rotation of each node when a unit load is exerted to neighboring members. Next, the nodal flexibility and anticipated stiffness matrix are calculated. Finally, by implementing work-equal force method and comparing the equivalent nodal force equations, shape functions are derived. Unlike the stiffness method which satisfies equilibrium equations only in certain integration points inside the elements, flexibility method guarantees that equilibrium equations are satisfied at all interior points of the element. Until now many papers are devoted to implement this technique in both static and dynamic analyses of straight or curved beams [33-35], which shows the flexibility of BDFs to cover a broad range of mechanical phenomenon.

Although obtaining closed-form solutions for in-plain and out-of-plain vibration of thin plates is worthwhile, due to different shape, boundary and loading conditions, finding a universal analytical solution for general plates' behaviour is not attainable. For this reason, numerical algorithms which are more flexible for solving differential equations of motion consisting coordinate - time variables are appealing to academia. In this paper, a new nine-node Lagrangian element was developed to analyse thin plates with varying cross-sections using the shape functions obtained for non-prismatic straight beams with minimum number of elements. It is believed that introducing this flexibilitybased element can facilitate further development of more efficient and accurate finite element models.

\section{METHODS AND MATERIALS}

\section{Basic displacement functions}

BDFs are mathematical functions derived from fundamental mechanical concepts. The detailed procedure to extract BFDs is provided by attarnejad [33], and an interested reader is encouraged to refer to abovementioned technical paper for further discussion over the methodology, which is the backbone of current research. Having said that, for clarification purposes, a brief description of the underlying assumptions is provided herein.

To clarify the definition of BDFs, consider a beam with one free node and the others clamped. A BDF can be described as the nodal displacement of the free node due to the unit load applied at distance ${ }^{x}$. For a three-node beam, BDFs can be defined as:

$b_{w m}$ : Vertical displacement of the $m$ th node due to the unit load at distance $x$ when the beam is clamped at the other nodes. 
$b_{\theta m}$ : Rotation angle of the $m$ th node due to the unit load at distance ${ }^{x}$ when the beam is clamped at the other nodes.

(Where $\mathrm{m}=1,2,3$ )

The visual description of $b_{w 1}, b_{\theta 1}, b_{w 2}, b_{\theta 2}, b_{w 3}, b_{\theta 3}$ are given in Figure 1 .

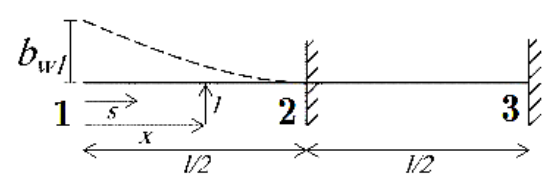

(a)

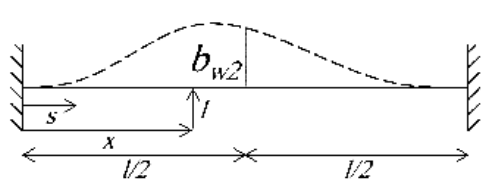

(c)

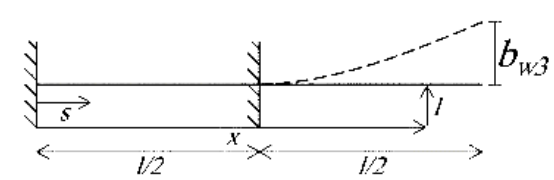

(e)



(b)

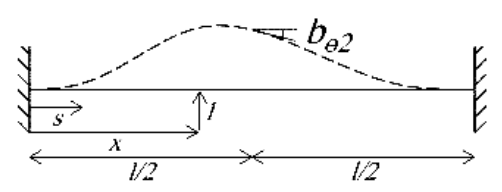

(d)

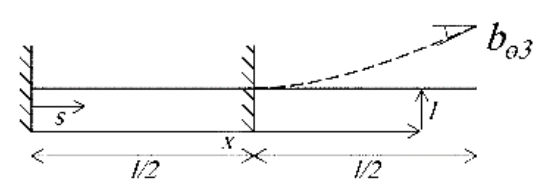

(f)

Figure 1. Definitions of basic displacement functions

\section{Nodal flexibility matrices}

According to the definition of nodal flexibilities and the equivalent definitions of BDFs, rotation, and curvature are the first and second derivative of BFDs, respectively. Therefore, nodal flexibility matrices can be shown as:

$$
\begin{aligned}
& \mathbf{F}_{\mathbf{1 1}}=\left[\begin{array}{cc}
b_{w 1}(0) & b_{\theta 1}(0) \\
\left.\frac{d b_{w 1}}{d x}\right|_{x=0} & \left.\frac{d b_{\theta 1}}{d x}\right|_{x=0}
\end{array}\right] \\
& \mathbf{F}_{22}=\left[\begin{array}{cc}
b_{w 2}\left(\frac{l}{2}\right) & b_{\theta 2}\left(\frac{l}{2}\right) \\
\left.\frac{d b_{w 2}}{d x}\right|_{x=\frac{l}{2}} & \left.\frac{d b_{\theta 2}}{d x}\right|_{x=\frac{l}{2}}
\end{array}\right] \\
& \mathbf{F}_{33}=\left[\begin{array}{cc}
b_{w 3}(l) & b_{\theta 3}(l) \\
\left.\frac{d b_{w 3}}{d x}\right|_{x=l} & \left.\frac{d b_{\theta 3}}{d x}\right|_{x=l}
\end{array}\right]
\end{aligned}
$$

Moreover, the stiffness matrix is calculated by inverting the nodal flexibility matrix. 


\section{New shape functions}

Consider an Euler-Bernoulli beam with variable thickness clamped at its two ends as well as its middle part. The beam is subjected to an external general loading as shown in Figure 2. To simplify the analysis, the beam was decomposed into two isostatic cantilever configurations with loading and reaction forces.


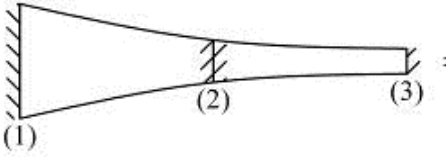

(a)

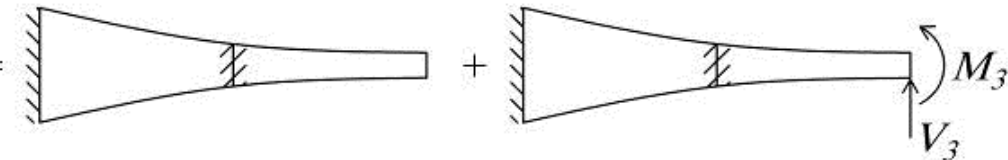

(b) (c)

Figure 2. General non-prismatic beam divided into two configurations (b) and (c)

In configuration (b), according to BDFs definitions, the nodal displacement of point (3) due to the external load can be calculated as follows:

$$
\left\{\begin{array}{l}
w_{3} \\
\theta_{3}
\end{array}\right\}^{(b)}=\int_{l} q(x)\left\{\begin{array}{l}
b_{w 3} \\
b_{\theta 3}
\end{array}\right\} d x
$$

In configuration (c), the nodal displacement of point (3) can be calculated using flexibility matrix:

$$
\left\{\begin{array}{l}
w_{3} \\
\theta_{3}
\end{array}\right\}^{(c)}=\mathbf{F}_{\mathbf{3 3}}\left\{\begin{array}{l}
V_{3} \\
M_{3}
\end{array}\right\}
$$

By imposing boundary conditions for the displacement of point (3) we have:

$$
\left\{\begin{array}{l}
w_{3} \\
\theta_{3}
\end{array}\right\}=\left\{\begin{array}{l}
w_{3} \\
\theta_{3}
\end{array}\right\}^{(b)}+\left\{\begin{array}{l}
w_{3} \\
\theta_{3}
\end{array}\right\}^{(c)}=\left\{\begin{array}{l}
0 \\
0
\end{array}\right\}
$$

Substituting equations (2) and (3) into equation (4) results in the reaction forces at point 3:

$$
\left\{\begin{array}{l}
V_{3} \\
M_{3}
\end{array}\right\}=-\mathbf{K}_{33} \int_{l} q(x)\left\{\begin{array}{l}
b_{w 3} \\
b_{\theta 3}
\end{array}\right\} d x
$$

Following a similar procedure, the reaction forces at points (1) and (2) are obtained as:

$$
\left\{\begin{array}{l}
V_{1} \\
M_{1}
\end{array}\right\}=-\mathbf{K}_{11} \int_{l} q(x)\left\{\begin{array}{l}
b_{w 1} \\
b_{\theta 1}
\end{array}\right\} d x
$$




$$
\left\{\begin{array}{l}
V_{2} \\
M_{2}
\end{array}\right\}=-\mathbf{K}_{22} \int_{l} q(x)\left\{\begin{array}{l}
b_{w v 2} \\
b_{\theta 2}
\end{array}\right\} d x
$$

Furthermore, the nodal equivalent loads, which are the equal and opposite responses to the reactions of supports are computed as:

$$
\mathbf{F}=\mathbf{G} \int_{l} q(x) \mathbf{b} d x
$$

Where $\mathbf{b}$ is a vector containing BDFs. and $\mathrm{G}$ is defined as:

$$
\mathbf{G}=\left[\begin{array}{lll}
\mathbf{K}_{11} & & \\
& \mathbf{K}_{22} & \\
& & \mathbf{K}_{33}
\end{array}\right]
$$

Employing work-equivalent force method, nodal forces are given by:

$$
\mathbf{F}=\int_{l} q(x) \mathbf{N}^{T} d x
$$

Shape functions can be obtained by comparing equations (7) and (9):

$$
\mathrm{N}=\mathrm{b}^{\mathrm{T}} \cdot \mathrm{G}
$$

\section{The proposed none-node element}

With regard to the three-node element formulations presented for the beam in one direction, the novel nine-node Lagrangian element for the plates in two directions can be shown in Figure 3. BDFs for plates are calculated by multiplying BDFs in two perpendicular directions using the theory discussed by Bogner et al. [36]. The multiplied shape functions are presented in Table 1 .

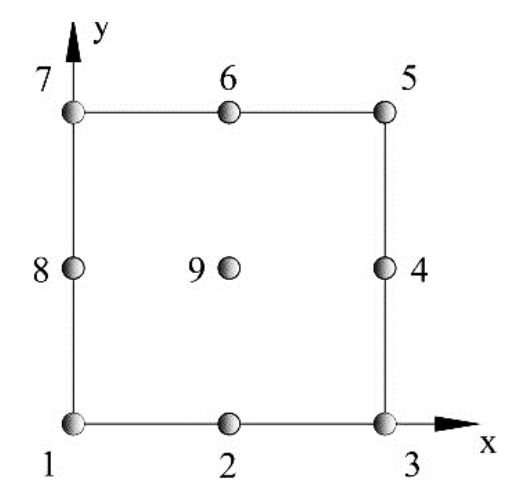

Figure 3. The nine-node Lagrangian element

Table 1. Definition of BDFs for plates

\begin{tabular}{|l|l|l|}
\hline$b_{1}=b_{w 1}(x) b_{w 1}(y)$ & $b_{2}=b_{\theta 1}(x) b_{w 1}(y)$ & $b_{3}=b_{w 1}(x) b_{\theta 1}(y)$ \\
\hline
\end{tabular}




\begin{tabular}{|l|l|l|}
\hline$b_{4}=b_{w 2}(x) b_{w 1}(y)$ & $b_{5}=b_{\theta 2}(x) b_{w 1}(y)$ & $b_{6}=b_{w 2}(x) b_{\theta 1}(y)$ \\
\hline$b_{7}=b_{w 3}(x) b_{w 1}(y)$ & $b_{8}=b_{\theta 3}(x) b_{w 1}(y)$ & $b_{9}=b_{w 3}(x) b_{\theta 1}(y)$ \\
\hline$b_{10}=b_{w 3}(x) b_{w 2}(y)$ & $b_{11}=b_{\theta 3}(x) b_{w 2}(y)$ & $b_{12}=b_{w 3}(x) b_{\theta 2}(y)$ \\
\hline$b_{13}=b_{w 3}(x) b_{w 3}(y)$ & $b_{14}=b_{\theta 3}(x) b_{w 3}(y)$ & $b_{15}=b_{w 3}(x) b_{\theta 3}(y)$ \\
\hline$b_{16}=b_{w 2}(x) b_{w 3}(y)$ & $b_{17}=b_{\theta 2}(x) b_{w 3}(y)$ & $b_{18}=b_{w 2}(x) b_{\theta 3}(y)$ \\
\hline$b_{19}=b_{w 1}(x) b_{w 3}(y)$ & $b_{20}=b_{\theta 1}(x) b_{w 3}(y)$ & $b_{21}=b_{w 1}(x) b_{\theta 3}(y)$ \\
\hline$b_{22}=b_{w 1}(x) b_{w 2}(y)$ & $b_{23}=b_{\theta 1}(x) b_{w 2}(y)$ & $b_{24}=b_{w 1}(x) b_{\theta 2}(y)$ \\
\hline$b_{25}=b_{w 2}(x) b_{w 2}(y)$ & $b_{26}=b_{\theta 2}(x) b_{w 2}(y)$ & $b_{27}=b_{w 2}(x) b_{\theta 2}(y)$ \\
\hline
\end{tabular}

The nine nodal flexibility matrices can be calculated using the BDFs. As an example $\mathrm{F}_{11}$, $\mathrm{F}_{55}$, and $\mathrm{F}_{99}$ matrices are calculated using the following matrices:

$$
\begin{aligned}
& \mathbf{F}_{11}=\left[\begin{array}{lll}
\left.b_{w 1}(x) b_{w 1}(y)\right|_{x=y=0} & \left.b_{\theta 1}(x) b_{w 1}(y)\right|_{x=y=0} & \left.b_{w 1}(x) b_{\theta 1}(y)\right|_{x=y=0} \\
\left.\frac{d b_{w 1}(x)}{d x} b_{w 1}(y)\right|_{x=y=0} & \left.\frac{d b_{\theta 1}(x)}{d x} b_{w 1}(y)\right|_{x=y=0} & \left.\frac{d b_{w 1}(x)}{d x} b_{\theta 1}(y)\right|_{x=y=0} \\
\left.\frac{d b_{w 1}(y)}{d y} b_{w 1}(x)\right|_{x=y=0} & \left.\frac{d b_{w 1}(y)}{d y} b_{\theta 1}(x)\right|_{x=y=0} & \left.\frac{d b_{\theta 1}(y)}{d y} b_{w 1}(x)\right|_{x=y=0}
\end{array}\right] \\
& \mathbf{F}_{55}=\left[\begin{array}{ccc}
\left.b_{w 3}(x) b_{w 3}(y)\right|_{x=y=l} & \left.b_{\theta 3}(x) b_{w 3}(y)\right|_{x=y=l} & \left.b_{w 3}(x) b_{\theta 3}(y)\right|_{x=y=l} \\
\left.\frac{d b_{w 3}(x)}{d x} b_{w 3}(y)\right|_{x=y=l} & \left.\frac{d b_{\theta 3}(x)}{d x} b_{w 3}(y)\right|_{x=y=l} & \left.\frac{d b_{w 3}(x)}{d x} b_{\theta 3}(y)\right|_{x=y=l} \\
\left.\frac{d b_{w 3}(y)}{d y} b_{w 3}(x)\right|_{x=y=l} & \left.\frac{d b_{w 3}(y)}{d y} b_{\theta 3}(x)\right|_{x=y=l} & \left.\frac{d b_{\theta 3}(y)}{d y} b_{w 3}(x)\right|_{x=y=l}
\end{array}\right] \\
& \mathbf{F}_{99}=\left[\begin{array}{ccc}
\left.b_{w 2}(x) b_{w 2}(y)\right|_{x=y=\frac{l}{2}} & \left.b_{\theta 2}(x) b_{w 2}(y)\right|_{x=y=\frac{l}{2}} & \left.b_{w 2}(x) b_{\theta 2}(y)\right|_{x=y=\frac{l}{2}} \\
\left.\frac{d b_{w 2}(x)}{d x} b_{w 2}(y)\right|_{x=y=\frac{l}{2}} & \left.\frac{d b_{\theta 2}(x)}{d x} b_{w 2}(y)\right|_{x=y=\frac{l}{2}} & \left.\frac{d b_{w 2}(x)}{d x} b_{\theta 2}(y)\right|_{x=y=\frac{l}{2}} \\
\left.\frac{d b_{w 2}(y)}{d y} b_{w 2}(x)\right|_{x=y=\frac{l}{2}} & \left.\frac{d b_{w 2}(y)}{d y} b_{\theta 2}(x)\right|_{x=y=\frac{l}{2}} & \left.\frac{d b_{\theta 2}(y)}{d y} b_{w 2}(x)\right|_{x=y=\frac{l}{2}}
\end{array}\right]
\end{aligned}
$$


Using the acquired nodal flexibility matrices and applying boundary conditions at the first node, the nodal loads associated with the external force $p$ can be obtained as follows:

$$
\left\{\begin{array}{l}
F^{1} \\
M_{x}^{1} \\
M_{y}^{1}
\end{array}\right\}=-\mathbf{F}_{\mathbf{1 1}}^{-\mathbf{1}} \int_{0}^{a} \int_{0}^{b} p_{z}(x, y) \cdot\left\{\begin{array}{l}
b_{w 1}(x) b_{w 1}(y) \\
b_{\theta 1}(x) b_{w 1}(y) \\
b_{w 1}(x) b_{\theta 1}(y)
\end{array}\right\} d x d y
$$

The same procedure can be applied to all the other nodes. Employing the equations obtained from nodal forces in elements, nodal equivalent loads for the elements can be presented as:

$$
[F]=-[\Sigma] \int_{0}^{a} \int_{0}^{b} p_{z}(x, y) \cdot[b] d x d y
$$

In which:



On the other hand, according to work-equivalent load method, nodal equivalent forces due to external load $q$ is defined as:

$$
F_{i}=\iint_{A} q(x, y) N_{i} d x d y
$$

By comparing Equations (13) and (15) discussed for the nodal forces in finite element method, the following equation would be obtained:

$$
[N]=[b]^{T} \cdot[\Sigma]
$$

In the above Equation, the vector $[b]=\left[b_{1} b_{2} b_{3} \ldots b_{27}\right]$ is the same as the one presented in Table 1, which contains BDFs. Combining Equation (16) with what was discussed for stiffness matrix method, leads to constructing the stiffness and consistent mass matrices for plates with variable cross section: 


$$
[K]=[\Sigma] .\left(\int_{0}^{a} \int_{0}^{b}\left[b^{\prime \prime}\right]^{T}[D]\left[b^{\prime \prime}\right] d x d y\right) \cdot[\Sigma]
$$

In which:

$$
\begin{gathered}
{\left[b^{\prime \prime}\right]^{T}=\left\{\begin{array}{c}
\frac{\partial^{2}}{\partial x^{2}} \\
\frac{\partial^{2}}{\partial y^{2}} \\
2 \frac{\partial^{2}}{\partial x \partial y}
\end{array}\right\} \cdot[b]} \\
{[D]=\frac{E(t(x, y))^{3}}{12\left(1-v^{2}\right)}\left[\begin{array}{ccc}
1 & v & 0 \\
v & 1 & 0 \\
0 & 0 & \frac{(1-v)}{2}
\end{array}\right]}
\end{gathered}
$$

The mass matrix required for dynamic analyses can be defined as:

$$
[M]=[\Sigma] .\left(\int_{0}^{a} \int_{0}^{b}[b]^{T} \rho h(x, y)[b] d x d y\right) \cdot[\Sigma]
$$

In these equations, $t$ represents thickness, $E$ elasticity modulus, $\rho$ mass density, $h(x, y)$ thickness, and $v$ Poisson's ratio.

\section{NUMERICAL EXAMPLES}

In the following sections, the accuracy and the reliability of the proposed element are verified for both static and free vibration analyses through several numerical examples.

\section{Static analysis of a simply supported square plate}

The first example consists a simply supported thin plate with constant thickness. The geometry of the plate is shown in Figure 4. Furthermore, the following geometrical and mechanical properties have been assumed (Table 2):

Table 2. Geometrical and material properties of the simply supported square plate

\begin{tabular}{ccccc}
\hline $\begin{array}{c}\text { Elasticity } \\
\text { Modulus } \\
\left(\mathrm{N} / \mathrm{m}^{2}\right)\end{array}$ & $\begin{array}{c}\text { Poisson's ratio } \\
(\mathrm{v})\end{array}$ & Thickness (m) & $\begin{array}{c}\text { Plate } \\
\text { dimension }(\mathrm{m})\end{array}$ & $\begin{array}{c}\text { Density } \\
\left(\mathrm{kg} / \mathrm{m}^{3}\right)\end{array}$ \\
\hline $2 \times 10^{8}$ & 0.3 & 0.01 & 1 & 8000 \\
\hline
\end{tabular}




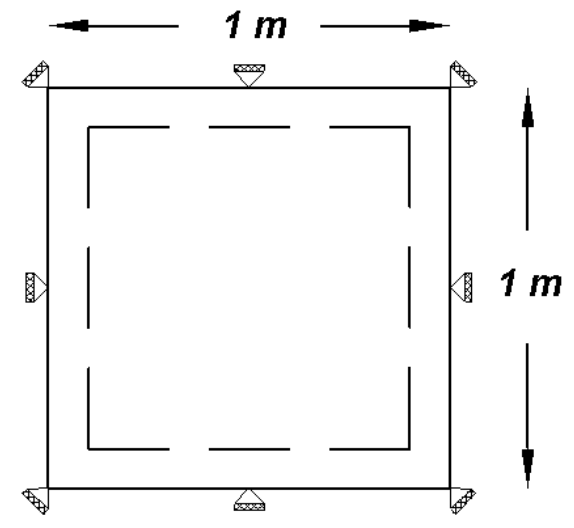

Figure 4. The simply supported rectangular plate

A 10-N concentrated load is applied at the center of the plate. The results of the analysis and the comparisons made with the previous studies are shown in Table 3.

Table 3. Non-dimensional deflections at the center of the rectangular plate

\begin{tabular}{lllll}
\hline Mesh & $\begin{array}{l}\text { Num. } \\
\text { Elements }\end{array}$ & $\begin{array}{l}\text { New } \\
\text { element }\end{array}$ & $\begin{array}{l}\text { Razaqpur et } \\
\text { al. [31] }\end{array}$ & $\begin{array}{l}\text { Belounar and } \\
\text { Guenfound [37] }\end{array}$ \\
\hline $2 \times 2$ & 4 & 0.0115478 & 0.012898 & 0.001828 \\
$4 \times 4$ & 16 & 0.0115951 & 0.011872 & 0.008449 \\
$8 \times 8$ & 64 & 0.0116088 & 0.011589 & 0.011164 \\
$16 \times 16$ & 256 & 0.0116056 & 0.011624 & 0.011374 \\
& \multicolumn{4}{c}{ Exact solution [38]: 0.0116 } \\
\hline
\end{tabular}

As is observed, the results obtained by implementing the new element is an acceptable approximation of the exact solution even with a few number of elements as four. This is largely based on the fact that the exact static deformations are used in deriving the BDFs. Nonetheless, by increasing the number of elements, the discrepancy observed between the obtained results and the exact solution was reduced. In most referenced solutions, a larger number of elements is required to guarantee a more convenient solution. For a better comparison, the error margins and the speed of convergence relative to the closedform solution are demonstrated in Figure 5. As can be seen, for a course mesh, the relative error in calculating out-of-plain deflection was reduced from more than $80 \%$ to less than $5 \%$ in case of implementing the new element. This speaks volumes about how efficient using this new element would be when restrictions are applied on the number of allowable elements in FEM analysis. 


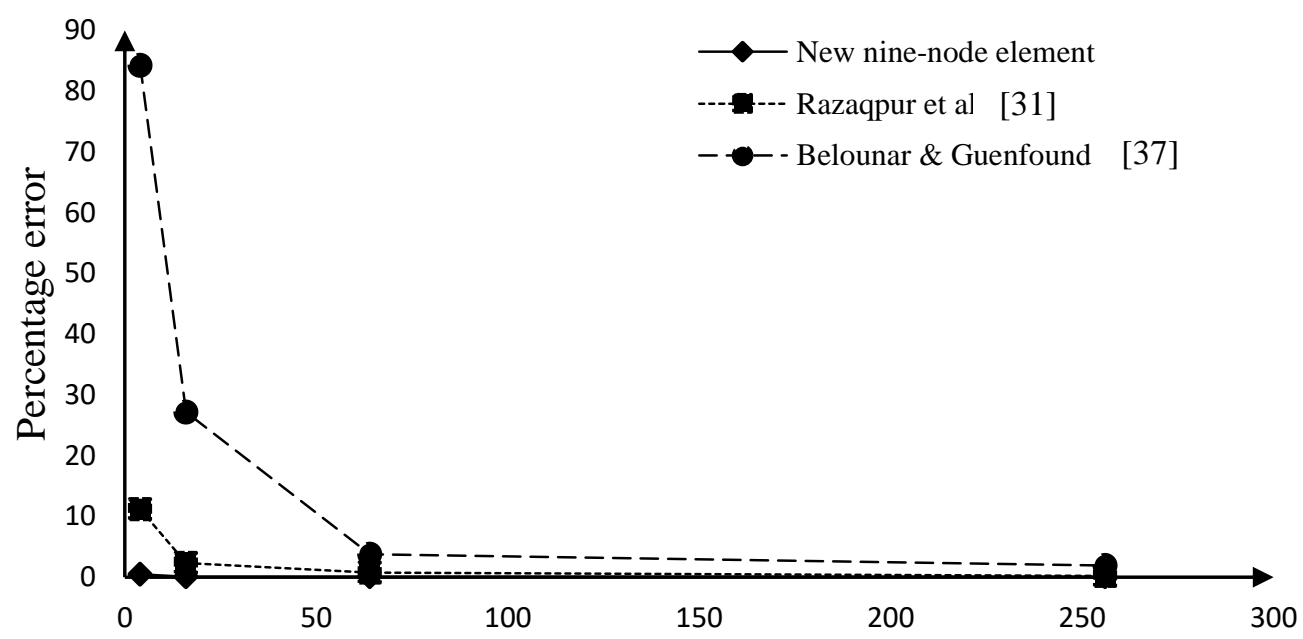

Number of elements

Figure 5. Percentage error in calculating the deflection of the simply supported square plate with constant thickness

For the second example, consider two plates with thickness variations according to Figure 6. The plates are simply supported at their four edges. Moreover, material characteristics is similar to that of the former example.

Variation in the thickness of the plates in Y direction is assumed based on two different scenarios: linear and quadratic. The equations governing this variation in thickness are given by:

$$
\begin{aligned}
& h_{l}(y)=h_{0}\left[1+\lambda f_{1}(y)\right]=0.01\left[1+0.2\left(2\left(\frac{y}{b}\right)-1\right)\right] \\
& h_{q}(y)=h_{0}\left[1+\lambda f_{1}(y)^{2}\right]=0.01\left[1+0.2\left(2\left(\frac{y}{b}\right)-1\right)^{2}\right]
\end{aligned}
$$

In the above equations, $h_{1}$ and $h_{q}$ are the thickness of the plates with linear and quadratic thickness variations, respectively. Furthermore, the value of $0.01 \mathrm{~m}$ is assigned to $\mathrm{h}_{0}$, which is the constant reference thickness value as depicted in Figure 6. Finally, the parameter $\lambda$ is taken as 0.2 according to the referred technical paper [39].

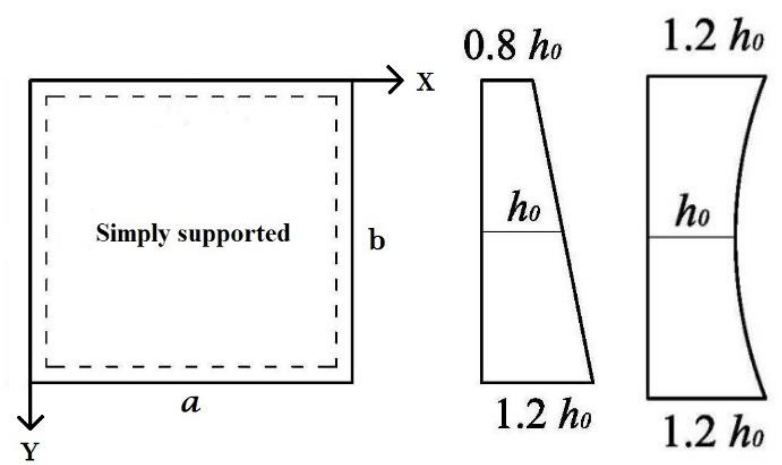

Figure 6. Linear and second-order variations in the thickness of the plates 
A $10 \mathrm{~N} / \mathrm{m}^{2}$ uniform load is applied to the plates and the static analysis is carried out for both models. The outcome of the analysis is shown for the linear and quadratic varying thickness plates in Tables 4 and 5, respectively. The criterion for verifying the results is the dimensionless out-of-plane displacement of the central node of each model. By comparing the results with the closed-form solution developed for rectangular plates under static loading [37], and also the displacements obtained by finite element analysis using reduced integrated four-noded shell element, it can be concluded that by employing a minimum number of elements the competency of the proposed element is verified. Moreover, for the new element, the convergence pattern to the closed-formed solution is different from those obtained from FEM. By increasing the number of elements in the finite element approach, the stiffness decreases which explains the rise in the output displacements. Conversely, BDFs which are based on the flexibility method result in a reduction in the displacements (stiffness increase) after each increase in the number of elements.

Table 4. Non-dimensional displacement at center of the plate with linear thickness variation $\left(w_{c} D / q a^{4}\right)$

\begin{tabular}{llll}
\hline Mesh & Number of elements & New element & FEM \\
\hline $2 \times 2$ & 4 & 0.4164 & 0.3703 \\
$4 \times 4$ & 16 & 0.4118 & 0.4111 \\
$8 \times 8$ & 64 & 0.4109 & 0.4111 \\
$16 \times 16$ & 256 & 0.4105 & 0.4116 \\
& Exact solution [37] $w_{c}=0.41 \times 10^{-2} \mathrm{qa}^{4} / \lambda D$ & \\
\hline
\end{tabular}

Table 5. Non-dimensional displacement at center of the plate with quadratic thickness variation $\left(w_{c} D / q a^{4}\right)$

\begin{tabular}{llll}
\hline Mesh & Number of elements & New element & FEM \\
\hline $2 \times 2$ & 4 & 0.3537 & 0.2762 \\
$4 \times 4$ & 16 & 0.3494 & 0.3405 \\
$8 \times 8$ & 64 & 0.3480 & 0.3479 \\
$16 \times 16$ & 256 & 0.3472 & 0.3502 \\
& Exact solution [37]: $w_{c}=0.3494 \times 10^{-2} q a^{4} / \lambda D$ \\
\hline
\end{tabular}

It should be noted that in the exact dimensionless solutions, $w_{c}$ is the displacement at the center point of the plates, $q_{0}$ is the uniform load, $a$ is the length of each side of square, and $D$ is defined as the flexural rigidity of the reference plate which is given by:

$$
D=\frac{E h_{0}^{2}}{12\left(1-v^{2}\right)}
$$

\section{Dynamic analysis of a tapered plate}

Take a four-edge simply supported plate with the following tabulated material and geometrical properties (Table 6): 
Table 6. Geometrical and material properties of the tapered plate

\begin{tabular}{lllll}
\hline $\begin{array}{l}\text { Elasticity } \\
\begin{array}{l}\text { Modulus } \\
\left(\mathrm{N} / \mathrm{m}^{2}\right)\end{array}\end{array}$ & $\begin{array}{l}\text { Poisson's ratio } \\
(\mathrm{v})\end{array}$ & $h_{0}(\mathrm{~m})$ & $\begin{array}{l}\text { Square plate } \\
\text { dimension }(\mathrm{m})\end{array}$ & $\begin{array}{l}\text { Density } \\
\left(\mathrm{kg} / \mathrm{m}^{3}\right)\end{array}$ \\
\hline $2 \times 10^{11}$ & 0.3 & 0.05 & 10 & 8000 \\
\hline
\end{tabular}

The linear thickness variation in the y direction can be calculated from the equation below:

$$
h_{y}(y)=0.05 \times(1+0.5 y)
$$

For the dynamic analysis of this plate, both the mass and stiffness matrices were extracted, and the first mode of natural frequency was calculated as shown in Table 7.

The natural frequencies obtained using the nine-node element is compared with those achieved by the two-dimensional transform method (2D-DTM) for thin plates with arbitrary varying thickness proposed by Semnani et al. [40]. According to the results, the natural frequencies reported here are increasing with a slow rate by an increase in the number of elements, and similar to previous examples, the number of employed elements is not a deciding factor for an appropriate approximation using the new element. Hence a course mesh consisting of 4 elements can results in a fine estimation of the natural frequency reported by [40].

Table 7. First mode natural frequencies of the simply supported plate $(\mathrm{rad} / \mathrm{s})$

\begin{tabular}{lll}
\hline Mesh & Num. of Elements & New element \\
\hline $2 \times 2$ & 4 & 48.1192 \\
$4 \times 4$ & 16 & 48.4959 \\
$6 \times 6$ & 36 & 48.5608 \\
$8 \times 8$ & 64 & 48.5912 \\
$16 \times 16$ & 256 & 48.6398 \\
$20 \times 20$ & 400 & 48.65041
\end{tabular}

Natural frequency obtained using 2D-DTM [40]: 47.138

\section{Discussion and conclusions}

In this paper a new method for analyzing thin plates with variable thickness using basic displacement functions was presented. For this purpose, a new nine-node Lagrangian element was introduced. The results of numerical examples from different sources were presented for a better comparison with proposed elements. The results revealed that in case of static analysis, with lower number of elements, more accurate results could be obtained using BDFs. Moreover, the speed of convergence to an acceptable solution greatly improved. It is worth mentioning that, the observed errors in the new element caused by the errors made in the calculation of BDFs unlike the errors observed in the FEM, which are mostly due to the assumed displacement fields. According to the results from the last numerical example, the proposed element was capable of producing acceptable results in the natural frequency analysis of plates with variable thickness. It worth mentioning that, the proposed nine-node plate element has been successfully applied to natural frequency analysis of tapered plates although the shape functions the 
element is grounded on are derived from static deformations. Finally, the number of elements needed for calculating the natural frequencies of plates with variable thickness in this method was much lower than the number of elements needed by finite element based solutions for the same or near accuracies. From a practical point of view, development of this new element, based on BDFs, may lead to more efficient approaches for structural analysis and could be expanded to solve a range of problems related to plates and shells with various geometries, loading and boundary conditions.

\section{REFERENCES}

[1] Ventsel E, Krauthammer T. Thin plates and shells. Marcel Dekker New York; 2001.

[2] Vijayakumar K. Review of a Few Selected Theories of Plates in Bending. International Scholarly Research Notices 2014;2014:1-9. doi:10.1155/2014/291478.

[3] Love AEH. The Small Free Vibrations and Deformation of a Thin Elastic Shell. Philosophical Transactions of the Royal Society A: Mathematical, Physical and Engineering Sciences 1888;179:491-546.

[4] Reissner E. On bending of elastic plates. Quarterly of Applied Mathematics 1947;5:55-68.

[5] Mindlin R. Influence of rotary inertia and shear on flexural motions of isotropic, elastic plates. Journal of Applied Mechanics 1951;18:31-8.

[6] Leissa, W. A. Vibration of plates. Ohio State Univeristy Columbus 1969.

[7] Nerantzaki MS, Katsikadelis JT. Buckling of plates with variable thickness - an analog equation solution. Engineering Analysis with Boundary Elements 1996;18:149-54.

[8] Eisenberger M, Alexandrov A. Buckling loads of variable thickness thin isotropic plates. Thin-Walled Structures 2003;41:871-89.

[9] Luong NTH, Tri TH. Influence of variable thickness on stability of rectangular plate under compression. Mechanics Research Communications 2005;32:139-46.

[10] Kobayashi H, Sonoda K. Buckling of Rectangular Plates with Tapered Thickness. Journal of Structural Engineering 1990;116:1278-89.

[11] Chen JT, Chen IL, Chen KH, Lee YT, Yeh YT. A meshless method for free vibration analysis of circular and rectangular clamped plates using radial basis function. Engineering Analysis with Boundary Elements 2004;28:535-45.

[12] Thai $\mathrm{CH}$, Nguyen TN, Rabczuk T, Nguyen-Xuan H. An improved moving Kriging meshfree method for plate analysis using a refined plate theory. Computers \& Structures 2016;176:34-49.

[13] Chen J-S, Hillman M, Chi S-W. Meshfree Methods: Progress Made after 20 Years. Journal of Engineering Mechanics 2017;143:04017001.

[14] Shu C, Wu WX, Ding H, Wang CM. Free vibration analysis of plates using leastsquare-based finite difference method. Computer Methods in Applied Mechanics and Engineering 2007;196:1330-43.

[15] Bert CW, Malik M. Free vibration analysis of tapered rectangular plates by differential quadrature method: a semi-analytical approach. Journal of Sound and Vibration 1996;190:41-63.

[16] Mohammed OH, Saeed MA. Numerical solution of thin plates problem via differential quadrature method using G-spline. Journal of King Saud University - 
Science 2018.

[17] Civalek Ö. A four-node discrete singular convolution for geometric transformation and its application to numerical solution of vibration problem of arbitrary straight-sided quadrilateral plates. Applied Mathematical Modelling 2009;33:300-14.

[18] Rajalingham C, Bhat RB, Xistris GD. Vibration of rectangular plates using plate characteristic functions as shape functions in the Rayleigh-Ritz method. Journal of Sound and Vibration 1996;193:497-509.

[19] Singhatanadgid P, Taranajetsada P. Vibration analysis of stepped rectangular plates using the extended Kantorovich method. Mechanics of Advanced Materials and Structures 2016;23:201-15.

[20] Shojaee S, Izadpanah E, Valizadeh N, Kiendl J. Free vibration analysis of thin plates by using a NURBS-based isogeometric approach. Finite Elements in Analysis and Design 2012;61:23-34.

[21] Rock T, Hinton E. Free vibration and transient response of thick and thin plates using the finite element method. Earthquake Engineering \& Structural Dynamics 1974;3:51-63.

[22] Brischetto S, Torre R. Exact 3D solutions and finite element 2D models for free vibration analysis of plates and cylinders. Curved and Layered Structures 2014;1.

[23] Kukreti AR, Farsa J, Bert CW. Fundamental Frequency of Tapered Plates by Differential Quadrature. Journal of Engineering Mechanics 1992;118:1221-38.

[24] Sakiyama T, Huang M. Free vibration analysis of rectangular plates with variable thickness. Journal of Sound and Vibration 1998;216:379-97.

[25] Ashour AS. Vibration of variable thickness plates with edges elastically restrained against translation and rotation. Thin-Walled Structures 2004;42:1-24.

[26] Kobayashi H, Sonoda K. Vibration and buckling of tapered rectangular plates with two opposite edges simply supported and the other two edges elastically restrained against rotation. Journal of Sound and Vibration 1991;146:323-37.

[27] Duan H, Gao S, Jiang B, Tan RCE. Analysis of a least-squares finite element method for the thin plate problem. Applied Numerical Mathematics 2009;59:97687.

[28] Qin QH. Hybrid Trefftz finite-element approach for plate bending on an elastic foundation. Applied Mathematical Modelling 1994;18:334-9.

[29] Han J-G, Ren W-X, Huang Y. A wavelet-based stochastic finite element method of thin plate bending. Applied Mathematical Modelling 2007;31:181-93.

[30] Armanios EA, Negm HM. An improved rectangular element for plate bending analysis. Computers \& Structures 1983;16:677-86. doi:10.1016/00457949(83)90117-7.

[31] Razaqpur AG, Nofal M, Vasilescu A. An improved quadrilateral finite element for analysis of thin plates. Finite Elements in Analysis and Design 2003;40:1-23.

[32] Reza Attarnejad. On the derivation of geometric stiffness and consistent mass matrices for non-prismatic Euler-Bernoulli beam elements. European Congress on Computational Methods in Applied Sciences and Engineering, Barcelona: 2000.

[33] Attarnejad R. Basic displacement functions in analysis of nonprismatic beams. Engineering Computations 2010;27:733-45.

[34] Attarnejad R, Shahba A, Semnani SJ. Analysis of Non-Prismatic Timoshenko Beams Using Basic Displacement Functions. Advances in Structural Engineering 2011;14:319-32.

[35] Attarnejad R, Shahba A. Basic displacement functions for centrifugally stiffened 
tapered beams. International Journal for Numerical Methods in Biomedical Engineering 2010;27:n/a-n/a.

[36] Bogner FK. The generation of interelement-compatible stiffness and mass matrices by the use of interpolation formulas. the First Conference on Matrix Methods in Structural Mechanics, Ohio: Wright-Patterson AFB; 1965, p. 397443.

[37] Belounar L, Guenfoud M. A new rectangular finite element based on the strain approach for plate bending. Thin-Walled Structures 2005;43:47-63.

[38] Timoshenko SP, Woinowsky-Krieger S. Theory of plates and shells. McGrawhill; 1959.

[39] Zenkour AM. An exact solution for the bending of thin rectangular plates with uniform, linear, and quadratic thickness variations. International Journal of Mechanical Sciences 2003;45:295-315.

[40] Semnani SJ, Attarnejad R, Firouzjaei RK. Free vibration analysis of variable thickness thin plates by two-dimensional differential transform method. Acta Mechanica 2013;224:1643-58. 